\title{
LA DIMENSIÓN INFORMACIONAL DE LA COMPETENCIA DIGITAL DE LOS PREADOLESCENTES. UN ESTUDIO DE CASOS
}

\author{
Esther Vila-Couñago ${ }^{1}$, Fernando Fraga-Varela² y Esther Martínez-Piñeiro ${ }^{3}$ \\ Universidade de Santiago de Compostela, España \\ 1esther.vila@usc.es; 2fernando.fraga@usc.es; ${ }^{3}$ esther.martinez@usc.es
}

\begin{abstract}
Resumen. La búsqueda, valoración y selección de información es una competencia imprescindible para la ciudadanía de hoy, que se enfrenta diariamente a una multitud de datos y fuentes que debe seleccionar y gestionar en situaciones muy diversas. En este contexto presentamos parte de los resultados del proyecto "Competencia digital en estudiantes de educación obligatoria. Entornos sociofamiliares, procesos de apropiación y propuestas de e-inclusión", que pretende indagar en el proceso de construcción de la Competencia Digital de los preadolescentes. En concreto se presentan algunos resultados relativos al desarrollo de la dimensión informacional de esta competencia. En este estudio se emplea un diseño mixto explicativo en dos fases: la primera, cuantitativa, para la selección de los casos que participan posteriormente en la segunda fase, cualitativa -un estudio de casos múltiple-. Los resultados nos permiten conocer las limitaciones de los preadolescentes en el proceso de búsqueda, selección y valoración de la información; observamos que, si bien emplean diversos buscadores y localizan información, no llegan a seleccionarla adecuadamente, analizándola e interpretándola, ni a cotejar las fuentes consultadas. Como conclusión destaca la necesidad de reforzar el desarrollo de esta dimensión en la enseñanza primaria, que forma parte de una competencia considerada clave en nuestro sistema educativo.
\end{abstract}

Palabras clave: Competencia Digital; Competencia Informacional; Estudio de Casos; Diseño Mixto Explicativo.

\section{THE INFORMATIONAL DIMENSION OF THE DIGITAL COMPETENCE OF PRETEENS. A CASES STUDY}

\begin{abstract}
Search, assessment and information selection is an indispensable competence for nowadays citizens, which face daily selecting and managing a large multitude of data and data sources in many different situations. In this context, we present some of the results of the project "Digital competence in compulsory education students. Social and family environments, appropriation processes and inclusion proposals", which intends to investigate the building process of the digital competence in preteens. Specifically, some results related to the development of the informational dimension of this competence are presented. The study used an explanatory mixed design consisting of an initial quantitative phase for the selection of cases followed by a qualitative multiple case study. The results have allowed us to know the limitations of preteens in the process of searching, selecting and assessing information; since we have observed that, though they make good use of several browsers and find information, they don't select, analyze or interpret adequately this information and they don't check and collate its sources. As a conclusion, it stands out the necessity of strengthening the development of this dimension in the primary education, since it is part of a key competence of our educational system.
\end{abstract}

Keywords: Digital Competence; Informational Competence; Cases Study; Explanatory Mixed Design.

\section{INTRODUCCIÓN}

La búsqueda, valoración y selección de información es cada vez más una competencia imprescindible para la ciudadanía, que se enfrenta diariamente a una multitud de datos, fuentes y recursos que debe seleccionar y gestionar en situaciones y contextos muy diversos. A esto hay que añadir la necesidad de que se tome conciencia del valor de la información 
frente a su desvalorización por exceso (infoxicación) o la falsedad (fake news) (Gewerc \& Fraga-Varela, 2019). El dominio de herramientas digitales resulta clave para el acceso a la cultura, a los servicios ofrecidos por las instituciones sociales y para la participación ciudadana.

En nuestro sistema educativo, es a partir de la etapa de Educación Primaria donde la Competencia Digital (en adelante, $C D$ ) se ha incorporado como una de las ocho competencias básicas en el currículo estatal (LOE, 2006) y se ha mantenido como una de las siete competencias clave en la modificación de la Ley Orgánica actualmente en vigor (LOMCE, 2013).

Según la Recomendación del Parlamento Europeo y del Consejo de 18 de diciembre de 2006, "las competencias se definen como una combinación de conocimientos, capacidades y actitudes adecuadas al contexto" (p.13), entendiendo por competencias clave "aquéllas que todas las personas precisan para su realización y desarrollo personales, así como para la ciudadanía activa, la inclusión social y el empleo" (p.13). Atendiendo al marco de referencia europeo para desarrollar y comprender la CD de los ciudadanos, concretado en el Proyecto DigComp, la CD es definida como:

un conjunto de conocimientos, habilidades, actitudes, estrategias y valores que son puestos en acción cuando usamos las tecnologías y los medios digitales para realizar tareas, resolver problemas, comunicarse, tratar información, colaborar, crear y compartir contenidos y crear conocimiento, de forma efectiva, eficiente, apropiada, crítica, creativa, autónoma, flexible, ética y reflexiva para el trabajo, el ocio, la participación, el aprendizaje, la socialización, el consumo y el empoderamiento (Ferrari, 2013, p. 30).

DigComp presenta un modelo conceptual de la CD configurada por cinco dimensiones: Información, Comunicación, Creación de Contenidos, Seguridad y Resolución de Problemas; delimitando además 21 sub-competencias.

El modelo recoge un tratamiento sistemático de cada una de las sub-competencias identificadas, aportando definiciones; descriptores para tres niveles de competencia; ejemplos de los conocimientos, habilidades y actitudes relacionadas con la competencia; y ejemplos de cómo la competencia podría aplicarse a propósitos específicos, como el aprendizaje y el empleo. 
La dimensión que constituye el objeto de estudio del presente trabajo es la denominada "Información y alfabetización informacional", que implica identificar, localizar, recuperar, almacenar, organizar y analizar la información digital, evaluando su finalidad y relevancia; y está configurada por tres sub-competencias (Ferrari, 2013; Vuorikari, Punie, Carretero, \& Van den Brande, 2016):

- Navegación, búsqueda y filtrado de datos, información y contenido digital.

- Evaluación de datos, información y contenido digital.

- Tratamiento de datos, información y contenido digital.

Resulta de especial interés acercarse a la Competencia Informacional por su relevancia social y por la atención que recibe en el currículum de enseñanza primaria. En este sentido, partimos de estudios que ponen de manifiesto que estos aprendizajes son tenidos en cuenta en los documentos oficiales de los centros educativos, pero al no tener tanta proximidad -como sí tienen otras competencias- con las áreas de conocimiento impartidas, su articulación y desarrollo en los procesos de enseñanza no están asegurados (Alonso-Ferreiro, 2016; FragaVarela, \& Rodríguez-Groba, 2019). Así mismo tenemos en cuenta los resultados de otras investigaciones previas que ponen el nivel socioeconómico del alumnado en relación con su rendimiento académico y su nivel de alfabetización digital (Berkowitz, Moore, Avi, \& Benbenishty, 2016; Harwell, Maeda, Bishop, \& Xie, 2017; Scherer \& Siddiq, 2019).

La investigación que aquí se presenta forma parte de del proyecto CDEPI "Competencia digital en estudiantes de educación obligatoria. Entornos sociofamiliares, procesos de apropiación y propuestas de e-inclusión", coordinado por el grupo de investigación Stellae de la Universidad de Santiago de Compostela (USC) y financiado por el Ministerio de Economía y Competitividad-Fondos FEDER (EDU2015-67975-C3-1-P). Concretamente se enmarca dentro de la primera fase del proyecto, cuya principal pretensión era comprender el proceso de construcción de la $C D$ de los niños y niñas de doce años -momento en que finalizan la etapa de Educación Primaria- y conocer el papel que juegan en él la familia y el grupo de iguales.

Además de ello, el interés de esta primera fase se centraba en identificar indicadores relevantes del alumnado de $6^{\circ}$ curso de Educación Primaria para poder ser tomados como elementos de referencia de cara a la construcción de un instrumento de evaluación de la CD en la siguiente fase del proyecto. 


\section{OBJETIVOS}

Con este trabajo se pretende comprender el proceso de adquisición y desarrollo del área informacional de la $\mathrm{CD}$ en alumnado de $6^{\circ}$ curso de Educación Primaria, así como analizar el papel que en esta juega la familia, la escuela y el grupo de iguales.

\section{METODOLOGÍA}

A continuación, detallamos la metodología empleada que responde a la complejidad de los objetivos formulados y del objeto de estudio, que se concreta en un diseño mixto, donde se utiliza la técnica de encuesta en la primera fase y se desarrolla un estudio de casos en la segunda fase.

\subsection{Diseño mixto}

En función de la clasificación de Creswell y Plano Clark (2018), el diseño mixto empleado es de tipo secuencial explicativo, en el que en la primera parte se utiliza un método cuantitativo cuyos resultados son necesarios para llevar a cabo la segunda fase. Más concretamente (véase la Figura 1), este diseño se denomina -siguiendo a ambos autores- de "selección de casos" (p. 82), atendiendo a la función que cumple la fase cuantitativa, que es usada cuando el/la investigador/a necesita información cuantitativa para seleccionar los participantes de la posterior fase de naturaleza cualitativa, la que mayor peso tiene en nuestro estudio.

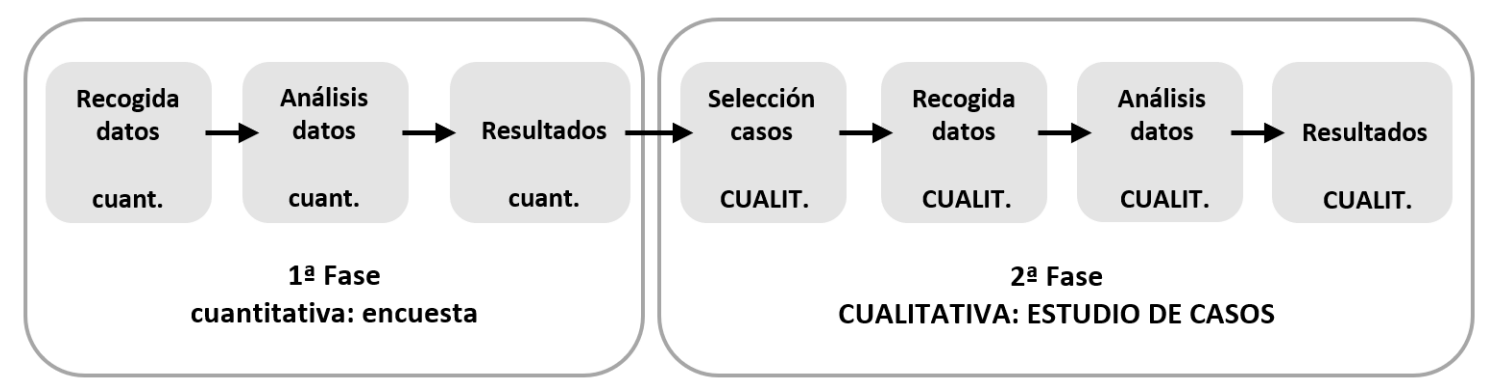

Figura 1. Diseño mixto explicativo de selección de casos.

\subsection{Estudio de casos}

Se optó por un estudio de casos múltiple, ya que este posibilita investigar "un fenómeno contemporáneo dentro de su contexto real de existencia" (Yin, 2003, p. 23), en el que participaron sujetos de distinta procedencia sociofamiliar. Se tuvieron en cuenta cuatro criterios de selección: alumnado de $6^{\circ}$ de Educación Primaria de escuelas que estén 
participando en proyectos de saturación tecnológica; representantes de entornos socioculturales y económicos diferentes; niños y niñas, atendiendo a la importancia que puede tener el género en este proceso de construcción de la CD, y que se presten a la investigación (criterio de accesibilidad). Se contó con la colaboración de centros escolares de la Comunidad Autónoma de Galicia, todos ellos inmersos en proyectos de saturación tecnológica, y se elaboró un cuestionario dirigido a las familias del alumnado de $6^{\circ}$ de primaria con el objetivo de atender a los restantes criterios. A través del instrumento elaborado ad hoc, en esa fase cuantitativa, se buscaba conocer las condiciones del entorno socio-familiar que permitiese seleccionar casos a partir de determinados indicadores (dispositivos tecnológicos existentes, estudios y profesión de los padres, y concesión de ayudas y bolsas, fundamentalmente).

Para este estudio en concreto se cuenta con tres casos (se utilizan nombres ficticios para garantizar el anonimato): dos niñas gemelas, Catarina y Lucía, pertenecientes a una familia acomodada, sin dificultades económicas y con un nivel sociocultural medio-alto; y un niño, Jaime, de un contexto familiar menos favorecido. En cada caso estudiado participaron distintos informantes, que permitieron la triangulación de los datos. A todos ellos se les garantizó el principio de consentimiento informado con base en los objetivos del estudio, el tipo de participación solicitada, la fuente de financiación del proyecto y los posibles usos de los resultados derivados. Se emplearon fundamentalmente dos técnicas cualitativas de recogida de datos:

- Entrevistas en profundidad, centradas básicamente en cinco dimensiones: familiahogar, aprendizaje, escuela, entorno social y autopercepción sobre el área informacional; adaptando el guion de los aspectos a tratar a cada uno de los informantes. Se realizaron cinco entrevistas a Jaime, dos a su madre y una a su profesora-tutora. Respecto a Catarina y Lucía, fueron tres las entrevistas realizadas a cada una, dos a sus progenitores y una a su profesora-tutora. Tuvieron lugar en espacios de la USC o de sus propios colegios y fueron grabadas en audio y transcritas de manera íntegra y literal.

- Observación participante de los sujetos del caso durante el desarrollo de las entrevistas. En este tiempo realizaron tareas escolares o jugaron a distintas aplicaciones con su ordenador portátil o su tableta que fueron grabadas en vídeo para su análisis posterior. 
En esta fase del estudio se pretendía, a través de estas técnicas, acompañar a los preadolescentes en su día a día en contacto con las tecnologías, ya que entendíamos desde el primer momento que el desarrollo de estas habilidades y competencias no se produce en un solo espacio social, ni está restringido únicamente a los entornos formales de formación (Gewerc \& Fraga-Varela, 2019). Los distintos informantes y escenarios escogidos nos posibilitaban obtener información más rica y precisa de este proceso. La recogida de datos se llevó a cabo en el segundo y tercer trimestre del curso 2016-2017.

La información recogida se analizó con el apoyo del programa Atlas.ti (versión 7), siguiendo las pautas de la teoría fundamentada propuesta por Glaser y Strauss (1967). En una primera fase se realizó una codificación inductiva de una de las entrevistas por parte de cada uno de los miembros del equipo de investigación, con la posterior puesta en común, revisando los códigos obtenidos y acordando pautas para análisis posteriores. Luego, fueron dos los investigadores dedicados a codificar el material de cada caso, contrastando la codificación realizada entre ellos. De este análisis surgió un sistema de categorías que incluía los contextos en los cuales se desenvuelve la Competencia Informacional, las actividades concretas realizadas (de búsquedas y de evaluación de la información) y las opiniones sobre el grado de desarrollo de la referida competencia. En una fase posterior, se asociaron las categorías y subcategorías intentando encontrar claves en el texto que permitieran relacionarlas (García \& Manzano, 2010). El proceso de análisis finalizó con una devolución personalizada de los principales hallazgos, concretada en un informe, a cada una de las familias y a los centros educativos, que sirvió como estrategia de validación de las observaciones e interpretaciones efectuadas.

\section{LA COMPETENCIA INFORMACIONAL DE LOS PREADOLESCENTES}

Los resultados se presentan atendiendo a una contextualización de cada caso que permite conocer el entorno que rodea a los protagonistas y, a continuación, se exponen los distintos elementos que aparecen tras el análisis realizado sobre el área informacional de la CD, apoyándonos en citas y en aspectos observados y abordando los diferentes entornos donde se adquiere dicha competencia. 


\subsection{La Competencia Informacional de Jaime}

Jaime es un niño de 12 años, estudiante de $6^{\circ}$ curso de Educación Primaria en un centro público de una de las grandes ciudades de la Comunidad Autónoma de Galicia. Jaime repitió $4^{\circ}$ de Educación Primaria y presenta ciertas dificultades en ámbitos como las matemáticas o el lenguaje escrito. Además, el niño fue diagnosticado de Trastorno por Déficit de Atención e Hiperactividad (TDAH). Jaime vive en un piso (propiedad de su abuela materna), ubicado en la periferia de la ciudad. Jaime residía con su madre -que es empleada de hogar-, con su padre -que se encuentra en el paro- y con su hermana pequeña, pero durante el proceso de recogida de datos su entorno familiar sufrió importantes cambios debido al divorcio de sus progenitores. A raíz de la separación, el padre abandonó el domicilio familiar, por lo que actualmente en el piso conviven el niño, su madre y su hermana.

Jaime realiza un gran uso de YouTube en su tiempo de ocio, sobre todo a través de su tablet y el móvil de su madre. Sigue a varios youtubers: Makiman131, EIChurches, VEGETTA777, Makina, Nexxuz, eLCris1017. Los ha conocido a través de su padre o de la promoción que unos hacen de otros para conseguir suscriptores en YouTube. Algunos de estos youtubers, no solo muestran sus hazañas en los videojuegos, sino que también dan a conocer retazos de su vida, hacen bromas, proponen retos y crean sus propios raps, aunque Jaime prefiere claramente sus vídeos de videojuegos. Él, como jugador asiduo de videojuegos, consume vídeos en los que los youtubers graban y comparten sus partidas. Estos constituyen importantes fuentes de aprendizaje para Jaime.

Los resultados obtenidos nos muestran que una de las dimensiones de la CD más desarrolladas es precisamente la relativa a la "Información y alfabetización informacional". A lo largo de las entrevistas con él, constatamos que entiende perfectamente cómo se distribuye la información en los medios digitales, sabe cómo puede encontrar la información con los diferentes motores de búsqueda (Google, YouTube), utiliza palabras clave en sus consultas apoyándose en las opciones de autocompletar, sigue la información presentada a través de hipervínculos y muestra una actitud proactiva hacia la búsqueda de información en la red. Esto mismo lo ponen de relieve su madre y su tutora:

Madre: Él mismo, por ejemplo, cuando escuchas tú alguna palabra y no sabes lo que es: "déjame aquí el teléfono que vamos a buscar en el Google".

(Jaime; entrevista2_madre)

Tutora: [...] El manexa moi ben os buscadores, os navegadores, sabe manexar e tal. El entra nunha páxina web y la controla perfectamente, é dicir, cunha visualización que a 
outros rapaces lles custa máis pois entender que hai pestanas, que es pestanas, a onde che levan e tal... El iso o controla moi ben [...].

(Jaime; entrevista_tutora)

Se observa claramente que el desarrollo de esta dimensión viene promovido desde dos frentes. Por una parte, se fomenta a través de las actividades que Jaime realiza en la escuela con el ordenador del aula, sobre todo en las áreas lingüísticas (lengua castellana y lengua gallega). En este sentido, también se incluyen los deberes para casa, en los que las búsquedas de información las realiza con el teléfono móvil de la madre. Si bien, en ocasiones, las consultas las hace la propia madre y él simplemente copia. Y, por otra parte, esta área competencial se promueve a través del uso que hace de YouTube con su tablet buscando y consultando los vídeos de los youtubers para luego jugar a los videojuegos.

Jaime también valora positivamente su capacidad para buscar información, en especial respecto a sus compañeros y compañeras de clase. No obstante, aunque el dominio de Jaime es alto respecto a la navegación y búsqueda de información, advertimos que para los trabajos escolares no analiza ni interpreta la información que encuentra, ni tampoco coteja las fuentes consultadas. Se suele quedar con la información de una única página, sólo mira "en la primera que aparece siempre" (Jaime; entrevista3), que suele coincidir con la web de la Wikipedia. Normalmente subraya todo el contenido, copiándolo y pegándolo en otro documento. El único caso en el que Jaime revisa la información es para traducir de castellano a gallego.

Entrevistadora: Y, por ejemplo, imagínate, te mandan buscar información sobre..., sobre Minecraft, ¿no? Por ejemplo. Vale, ¿lo coges de una página?, ¿o buscas varias páginas? Jaime: De la Wikipedia.

Entrevistadora: De la Wikipedia. Y, luego, ¿qué haces con eso? Jaime: Después... Le pones, le..., pongo en azul todo lo que quiero, y le doy a copiar y después lo pego.

Entrevistadora: Vale. Lo pegas, y, ¿luego lo entregas así?, ¿o lo...?

Jaime: Sí.

Entrevistadora: ¿Sí? ¿O lo revisas? ¿O miras en otras páginas?

Jaime: $A$ veces cuando, por ejemplo, si está en gallego, hay palabras que están por ejemplo en castellano, las tengo que escribir en gallego.

Entrevistadora: Vale. O sea, tú traduces, ¿no?, entonces.

Jaime: Sí.

(Jaime; entrevista3) 


\subsection{La Competencia Informacional de Lucía y Catarina}

Catarina y Lucía forman parte de una familia acomodada, sin dificultades económicas. Viven en una urbanización a las afueras de una pequeña ciudad gallega con su madre, su padre, y su hermano mayor. Además, desde hace unos años, tras la muerte de su abuelo, su abuela materna también vive con ellos. Tanto su padre como su madre poseen estudios superiores y trabajan en el ámbito de la enseñanza. Ambas hermanas dedican su tiempo fuera del horario escolar a la formación musical y el deporte; las tecnologías ocupan en su ocio un papel secundario.

La búsqueda de información es una actividad que llevan a cabo habitualmente para la realización de tareas escolares utilizando el ordenador de casa, del colegio o el iPad. Tanto las niñas como los padres concuerdan en ello:

Entrevistadora: ¿Cuál es la información que más buscas?, para que...

Catarina: ¿Para ordenador? No sé, a lo mejor los deberes o...

(Catarina; entrevista1)

Madre: A veces consultan, también.

Padre: $O$ a veces consultan, hacen consultas. $Y$ en el nuestro también (se refieren a su ordenador).

Entrevistadora: ¿O sea, consultas por...?

Padre: Pues del cole...

(Catarina y Lucía; entrevista1_padres)

Las niñas aseguran realizar pocas búsquedas en la red vinculadas a su tiempo de ocio, centrándose en aspectos académicos para desarrollar trabajos escolares. Sí reconocen buscar algún programa de televisión, como algún capítulo de MasterChef que no hayan podido ver entero (al emitirse hasta horas muy tardías).

Parecen desconocer las posibles diferencias entre distintos motores de búsqueda y las posibilidades que puede ofrecer cada uno, así como el uso adecuado de los términos de búsqueda, que emplean de un modo fundamentalmente intuitivo. No son procesos que podamos definir como organizados o sistemáticos, no empleando palabras clave y accediendo al primer resultado de búsqueda que encuentran, sin utilizar criterios de selección.

Entrevistadora: [Risas] Y... y los dibujos, ¿dónde los buscas?

Catarina: En Internet, en...

Entrevistadora: ¿En dónde? ¿En dónde sueles buscar? 
Catarina: En cualquier lado que me aparezca, o en Google o... Busco dibujos fáciles.

(Catarina; entrevista3)

Entrevistadora: Sobre todo, ¿es lo que...? ¿Es lo que más usas o...? ¿O usas también otras...? Lucía: También, a ver... Utilizo... Todos utilizamos la Wikipedia porque la profe dice: "Buscad esto en la Wikipedia", por ejemplo...

Entrevistadora: Ah, ¿ya os lo dice la..., la profe?

Lucía: Sí, porque... No sé porque... Y... Pero para el inglés, para el vocabulario que no sabemos hay una página... ¿Te la busco? [Lucía abre una pestaña nueva, se sitúa en el buscador y teclea "que ver en yocohama". Espera a que salga un desplegable con distintas opciones y pincha en la primera de ellas].

(Lucía; entrevista2)

Cuando buscan para localizar aplicaciones lo hacen con un objetivo concreto porque previamente les han hablado de un juego específico; no manifiestan -según el padre- una actitud proactiva hacia la búsqueda de información que les lleve a explorar y encontrar nuevos contenidos o aplicaciones:

Padre: Pero, pero claro ellas normalmente cuando... Bueno, supongo que pasará con muchos, ¿no? No hacen una búsqueda activa de las cosas, es decir, a ver si hay algo... Por lo menos por lo que yo detecto, ellas llegan con la noticia de un juego o una aplicación.

(Catarina y Lucía; entrevista2_padres)

Cuando llevan a cabo alguna búsqueda para localizar un dato de interés para ellas, este no suele ser realmente relevante, según incide el padre al identificar o definir la información buscada por las niñas como de escaso valor o interés real: "Que lo que suelen consultar son chorradas" (Catarina y Lucía; entrevista2_padres).

Progenitores y maestra coinciden en la dificultad para seleccionar información pertinente en los niños y niñas de esta edad, ya que habitualmente se quedan con la primera información que obtienen. No obstante, tanto la madre como el padre aseguran tratar de enseñarles una serie de pautas para buscar y seleccionar la información con cierto criterio, contrastando fuentes y tratando de filtrar, analizar y transformar los datos en lugar de copiarlos miméticamente. 
Padre: El Google, el Google, sí, buscan en Google...

Madre: Yo creo que sí.

Entrevistadora: Sí, y copian, leen la respuesta y la copian.

Madre: A ver, yo le suelo decir: "no pongas todo exactamente igual, lo lees y luego lo interpretas un poco y lo pones a tu manera".

Entrevistadora: Claro, ¿porque cogen, por ejemplo, una página o buscan más...? Para como contrastar.

Padre: Mira, no sé, yo por la experiencia que tengo, tanto con ellas como con todos los niños a esos niveles lo que hacen es, primera cosa que aparece...

Madre: "Pum".

Padre: Yo les digo, siempre: "mirad en varias". Claro, algo que supone un trabajo ¿no?

(Catarina y Lucía, entrevista2_padres)

Tutora: Mira, o da selección da información non están moi preparados. O sea, son nenos que todavía non distinguen moito...

(Catarina y Lucía; entrevista_tutora)

A partir de esta creencia, de la dificultad de los niños de buscar y seleccionar adecuadamente la información, la profesora acostumbra a darles los enlaces de las páginas que deben consultar, sin facilitar el aprendizaje de buscar y seleccionar información.

Entrevistadora: Pero normalmente, por ejemplo, cuando quieres buscar una información o un dato, ¿sueles ir a la Wikipedia?

Catarina: No sé, donde nos diga la profe.

$[\ldots]$

Entrevistadora: Y tú la Wikipedia, la usas entonces normalmente.

Catarina: No, cuando lo dice la...

Entrevistadora: La profe.

(Catarina; entrevista2)

La maestra considera que ambas saben buscar mejor y más información que el resto de la clase porque "son nenas que se esforzan moito", polo que "Entón buscan máis información, a presentan mellor, se esmeran en colores, en fondos" (Catarina y Lucía; entrevista_tutora). Su competencia la relaciona claramente con su nivel académico: "Pero porque son dúas nenas de sobresaliente. En todo. Entón eso tamén se nota no traballo que fan" (Catarina y Lucía; entrevista_tutora). En lo relativo a la gestión y almacenamiento de datos y contenido digital, 
observamos en ambas hermanas cierta dificultad en encontrar un archivo que pensaban que estaba guardado:

Entrevistadora: Y, entonces, esas fotos, ¿dónde las tienes guardadas?

Lucía: Pues mira, me saco una foto y yo te la enseño.

Entrevistadora: Vale.

Lucía: [saca foto] [ruido] [silencio]

Entrevistadora: ¡Ah! Vale, vas a fotos.

Lucía: Mmmmmm. A ver, voy a mirar una cosa que se cambia la cara, pero no sé dónde está.

Entrevistadora: ¡Qué guay!

Lucía: No sé dónde está, aquí no. Mmmm. Aquí. Pero no sé dónde está, puede estar por aquí.

[Lucía continúa buscando la fotografía que se sacó anteriormente. Accede a todas las carpetas almacenadas en "galería" pero sigue sin encontrarla].

(Lucía; entrevista3)

El dispositivo preferente para almacenar la información es el pendrive. Acostumbran a guardar del ordenador a un pen o de una memoria al pen. Aprendieron a hacer copias en el pendrive en quinto curso, en el aula, aunque reconocen que no lo hacen casi nunca.

Observamos que ambas poseen una Competencia Informacional básica, con dificultad para hacer búsquedas complejas, sin distinción entre motores de búsqueda, con cierto dominio del almacenamiento y la recuperación, pero con escasa competencia para la selección y evaluación de la información.

\section{CONCLUSIONES}

Los estudios de caso nos han permitido constatar el mayor o menor desarrollo de la dimensión informacional de la CD de los preadolescentes; observando, preguntando y analizando los productos de sus actividades mediadas por TIC. Jaime, a pesar de sus notables dificultades académicas, demuestra un mayor grado de desarrollo de la dimensión informacional promovida a través de su tiempo de ocio centrado en los videojuegos y YouTube-en contraste con Lucía y Catarina, alumnas que destacan por su alto rendimiento académico en general, pero con escaso interés por las tecnologías, especialmente en su tiempo de ocio. No obstante, 
a pesar de las diferencias, en ninguno de los casos presentados llegan a seleccionar la información hallada, analizándola e interpretándola, ni a cotejar las fuentes consultadas.

La situación que evidencian los casos pone en duda el papel de la escuela como normalizador de las necesidades de formación que presenta el alumnado con independencia de su nivel socioeconómico. La situación de Lucía y Catarina muestra un alto nivel de rendimiento académico en aprendizajes básicos, en coherencia con su nivel socioeconómico, situación de la que participa la familia y también la escuela (Harwell, Maeda, Bishop, \& Xie, 2017). Una situación que se escapa con Jaime: la familia pone el foco en sus habilidades digitales, donde se muestra una aparente situación de control que no es tal, y sin embargo carece de competencias para salir adelante en el espacio escolar.

Sin embargo, vemos como en los tres casos, con independencia de su nivel socioeconómico, la dimensión de la Competencia Informacional es claramente mejorable. Falta un conocimiento profundo de los instrumentos disponibles de búsqueda de información, de sus lógicas y funcionamiento. El centro educativo refuerza la búsqueda, pero desde una perspectiva muy simple, claramente vinculada a una pequeña extensión de las tareas que se desarrollan en las áreas de conocimiento. Los casos evidencian la baja relación, incluso sorprendente, que existe entre el nivel socioeconómico y el nivel de alfabetización digital (Berkowitz, Moore, Avi, \& Benbenishty, 2016; Scherer \& Siddiq, 2019) y pone en entredicho el papel de la escuela. Los centros educativos incorporan en su documentación estos aprendizajes (Alonso-Ferreiro, 2016) porque los marcos legislativos obligan, pero en el caso de las competencias que no tienen equivalencias con las áreas de conocimiento, como la CD, se vacían de contenido y quedan sin trabajar, ya que no tiene ningún estándar u objetivo específico en los currículos que la vertebre de forma explícita (Fraga-Varela \& RodríguezGroba, 2019). Y las familias no hacen sino confirmarlo al trabajar lo que se solicita de forma explícita desde la escuela, relegando el trabajo de esta dimensión de la CD a un segundo lugar.

Estos resultados ponen de relieve la necesidad de seguir indagando en el desarrollo de la Competencia Informacional de los preadolescentes y del papel que juega la familia y la escuela como espacios clave para su aprendizaje. 


\section{REFERENCIAS}

Alonso-Ferreiro, A. (2016). Competencia digital y escuela: estudio de caso etnográfico en dos CEIP de Galicia (Tesis doctoral non publicada). Universidad de Santiago de Compostela, Santiago de Compostela.

Berkowitz, R., Moore, H., Astor, R. A., \& Benbenishty, R. (2017). A research synthesis of the associations between socioeconomic background, inequality, school climate, and academic achievement. Review of Educational Research, 87(2), 425-469. doi: https://doi.org/10.3102/0034654316669821

Creswell, J. W., \& Plano, V. L. (2018). Designing and conducting mixed methods research. (3 ${ }^{a}$ ed.). Thousand Oaks, CA: SAGE.

Ferrari, A. (2013). DIGCOMP: A Framework for Developing and Understanding Digital Competence in Europe. Sevilla: Joint Research Centre, Institute for Prospective Technological Studies. doi: https://doi.org/10.2788/52966

Fraga-Varela, F., \& Rodríguez-Groba, A. (2019). Bieito: ¿competente digital? Una responsabilidad de todos y de nadie. En A. Gewerc \& E. Martínez-Piñeiro (Coords.), Competencia digital y preadolescencia. Los desafíos de la e-inclusión. Madrid: Síntesis.

García Hernández, G. E., \& Manzano Caudillo, J. (2010). Procedimientos metodológicos básicos y habilidades del investigador en el contexto de la teoría fundamentada. Iztapalapa: Revista de Ciencias Sociales y Humanidades, 69, 17-39.

Gewerc, A., \& Fraga-Varela, F. (2019). Competencia digital e inclusión social: cuando las condiciones socioculturales se imponen. En A. Gewerc \& E. Martínez-Piñeiro (Coords.), Competencia digital y preadolescencia. Los desafíos de la e-inclusión (pp. 21-42). Madrid: Síntesis.

Glaser, B. G., \& Strauss, A. L. (1967). The Discovery of grounded theory: strategies for qualitative research. New York: Aldine Publishing. doi: https://doi.org/10.1097/00006199-196807000-00014

Harwell, M., Maeda, Y., Bishop, K., \& Xie, A. (2017). The surprisingly modest relationship between SES and educational achievement. The Journal of Experimental Education, 85(2), 197-214. doi: 10.1080/00220973.2015.1123668

Ley Orgánica 2/2006, de 3 de mayo, de Educación. BOE, 4 de mayo, núm. 106, pp. 17158-17207.

Ley Orgánica 8/2013, de 9 de diciembre, para la Mejora de la Calidad Educativa. BOE, 10 de diciembre, núm. 295, pp. $97858-97921$.

Recomendación del Parlamento Europeo y del Consejo de 18 de diciembre de 2006 sobre las competencias clave para el aprendizaje permanente (2006/962/CE). Diario Oficial de la Unión Europea, L 394, 10-18.

Scherer, R., \& Siddiq, F. (2019). The relation between students' socioeconomic status and ICT literacy: Findings from a meta-analysis. Computers \& Education, 138, 13-32. doi: https://doi.org/10.1016/j.compedu.2019.04.011

Vuorikari, R., Punie, Y., Carretero, S., \& Van den Brande, G. (2016). DigComp 2.0: The Digital Competence Framework for Citizens. Update Phase 1: The Conceptual Reference Model. Luxembourg Publication Office of the European Union. doi: https://doi.org/10.2791/11517

Yin, R. K. (2003). Case study research. Design and methods. (3ª ed.). Londres: SAGE. 